\title{
Eugenia Uniflora Active Fraction Inhibits Dalton Lymphoma Ascites in Swiss Albino Mice - An in Vivo Study
}

\author{
S. Syama ${ }^{a}$ \\ ${ }^{\text {a }}$ School of Biosciences, \\ Mahatma Gandhi University, Priyadarshini Hills, \\ Kottayam, Kerala, India.
}

\author{
Dr. M.S Latha \\ *Corresponding author: \\ School of Biosciences, Mahatma Gandhi University, \\ Priyadarshini Hills, Kottayam, Kerala, India.
}

\begin{abstract}
Cancer is one of the major leading causes of death. The cancer cells attack and demolish normal cells. The present study was designed to evaluate the anticancer activity of Eugenia uniflora active fraction (EF18) against Dalton lymphoma ascites tumor model. Eugenia uniflora active fraction was obtained from the partial purification of crude ethanolic extract of Eugenia uniflora and bioassay graded screening of the fractions obtained. In vitro cytotoxicity studies were conducted using short term trypan blue method. For the In vivo anti-cancer study in Dalton's lymphoma ascites, two doses of Eugenia uniflora active fraction $500 \mathrm{mg} / \mathrm{kg}$ and $250 \mathrm{mg} / \mathrm{kg}$ were taken. The doses were selected on the basis of acute toxicity studies. The anti-cancer activity was assessed by monitoring the tumor volume, percentage survival time, LDH (Lactate dehydrogenase enzyme) level and histopathological analysis. The standard drug used for In vivo study was Cyclophosphamide. This Cyclophosphamide is mainly used to treat a wide spectrum of cancers. In vitro studies showed that Eugenia uniflora active fraction exhibited cytotoxic activity. The results of the present in vivo study demonstrated that Eugenia uniflora active fraction, especially its higher dose $(500 \mathrm{mg} / \mathrm{kg})$ was better in reducing the tumor volume, increasing the percentage life span and lowering the LDH level when compared to the lower dose. The histopathological analysis also supported that Eugenia uniflora active fraction possessed anti-cancer activity. The outcome of the entire study showed that the higher dose of EF 18 was better than the lower dose.
\end{abstract}

Keywords-Dalton lymphoma; Trypan blue; Eugenia;Acute toxicity; Cyclophosphamide; anti-cancer.

\section{INTRODUCTION}

Cancer is the uncontrolled splitting of cells within a fraction. The cancer cells attack and demolish normal cells. Cancer acts as a burden in both the developing and developed countries [1]. Cancer is considered as the burning health issue and most threatening diseases [2]. Chemically derived drugs, chemotherapy, radiation are very common treatments of cancer. Both chemotherapy and radiation are lethal and toxic to normal cells. Most of these currently used anti- cancer drugs are harmful to the human health. Therefore new plant derived drugs are needed since they have fewer side effects [3].Cancer executes a serious burden on public health issues [4]. For the treatment of cancer, various secondary metabolites from plants were investigated. In some of the developing countries plants are used as the major source of treatment. In the human body, cancer can be characterized by the uncontrolled multiplication of cells [5]. Plants exhibited a major role in the history of cancer treatment. Herbal medicines play a significant role in human healthcare. Some chemical substances are responsible for the medicinal property of the plant [6]. Cancer remains one of the leading causes of morbidity and mortality globally. Cancer disturbs various cellular activities [7]. Cancer occurs as a result of uncontrolled bioenergetics metabolism and also by the overexpression of proto-oncogenes which in turn causes the liberalized cellular response. Carcinogenesis is a multistep process that involves intrinsic and extrinsic factors. The usage of substances to prevent the growth of carcinogenesis is known as chemoprevention. Cancer chemoprevention is also known as anti-carcinogenesis [8]. Obesity also results in the formation of uterine, breast, colon kidney and esophageal cancer. Usage of tobacco results in the lung, mouth, throat, stomach cancer. Causative agents of cancer include smoking, imbalances in the diet, hormonal imbalance, and some of the chronic inflammations [9].

Eugenia uniflora known as the Surinam cherry is taken for the study because of its therapeutic importances [10]. Disease in lymphocytes is known as lymphoma and this includes spleen, thymus and some lymphatic tissues. Dalton's Lymphoma ascites (DLA) are the transplantable tumors which grow rapidly. These tumors have aggressive behavior.

The main aim of the study was to determine the anticancer property of Eugenia uniflora active fraction (EF18) against Dalton lymphoma ascites tumor. For this in vitro short term cytotoxic studies and in vivo studies were carried out Normally cytotoxicity assays involve a short term exposure of transformed or cultured normal cells to a test sample substances in order to detect the toxicity potential. There are different parameters to detect the cytotoxicity among them trypan blue test is commonly used one to measure the cell membrane integrity [11] and also the cell viability. In this method the live cells are not coloured as they have intact membrane [12].In vivo anti-cancer studies of Eugenia uniflora active fraction (EF18) studies were carried out using Swiss Albino mice.Cyclophosphamide was used as the reference drug for the in vivo study. Cyclophosphamide is also used for treating autoimmune diseases. 


\section{MATERIALS AND METHODS}

\section{A. Preparation of crude ethanolic extract of Eugenia uniflora}

One of the commonly used extraction technique is the soxhlet method. For soxhlet extraction, the fresh leaves of Eugenia uniflora were collected, washed properly, then shade dried and powdered. For soxhlet extract, 50gm of Eugenia uniflora leaf powder was extracted in $500 \mathrm{ml}$ of the solvents. The solvents comprise petroleum ether, chloroform, ethyl acetate, methanol ethanol, and water. The solvents used for the soxhlet extraction were selected on the basis of their increasing polarity. Extraction was performed for about 72 hours. After 72 hours the extract and solvent remaining after evaporation in the round bottom flask were collected. The extraction procedure was continued successively until the required amount was attained. These solvent extracts were then filtered through Whatmann No.1 filter paper. The solvents collected are then evaporated to dryness using a rotary evaporator and stored at $40 \mathrm{C}$ in sterile vials. These dried Eugenia uniflora crude solvent extracts were taken for further studies. All these crude solvent extracts of Eugenia uniflora were screened for the most active crude solvent extract. Phytochemical analysis, the antioxidant evaluation revealed that the crude ethanolic extract of Eugenia uniflora was most active. This was then collected in large quantities and taken for further studies.

\section{B. Preparation of Eugenia uniflora active fraction}

Most active Eugenia uniflora fraction (EF18) was obtained after the partial purification using column chromatography technique and bioassay graded screening of crude ethanolic extract of Eugenia uniflora. This EF18 was used for in vitro and in vivo studies. The dosage of the EF18 was selected on the basis of acute toxicity studies. Two doses were taken for the study $500 \mathrm{mg} / \mathrm{kg}$ as a higher dose and $250 \mathrm{mg} / \mathrm{kg}$ as a lower dose. The dosage of Cyclophosphamide drug was $25 \mathrm{mg} / \mathrm{kg}$.

\section{C.Dalton's lymphoma ascites (DLA) Cell culture}

Dalton's lymphoma ascites (DLA) cells are maintained in Amala Cancer Research Centre, Thrissur. Dalton's lymphoma ascites cells were aspirated from the peritoneal cavity of the mice bearing the tumor and were then washed three times using PBS or normal saline. Viable suspension of $1 \times 10^{6}$ cells was then taken into tubes for further in vitro assays. For in vivo assays these cells are then injected into the intraperitoneal cavity of mice.

\section{Cytotoxicity assay using Trypan Blue method on Dalton's lymphoma ascites (DLA) cells}

The plant sample was studied for the short term in vitro cytotoxicity using the DLA by the Trypan Blue method [13] [14]. The tumor cells aspirated from the peritoneal cavity of tumor-bearing mice were washed thrice with PBS or normal saline. Cell viability was determined by trypan blue exclusion method. Viable cell suspension $\left(1 \times 10^{6}\right.$ cells in $0.1 \mathrm{ml}$ ) was added to the tubes containing various concentrations of the test compounds and the volume was made up to $1 \mathrm{ml}$ using phosphate-buffered saline (PBS). The control tube contained only cell suspension. These assay mixtures were incubated for 3 hours at $37^{\circ} \mathrm{C}$. The further cell suspension was mixed with $0.1 \mathrm{ml}$ of $1 \%$ trypan blue and kept for 2 to 3 minutes and loaded on a hemocytometer. Dead cells take up the blue color of the trypan blue while live cells do not take up the dye. The numbers of stained and unstained cells were counted separately.

$$
\% \text { Cytotoxicity }=\frac{\text { No: of dead cells }}{\text { No: of live cell+ No: of dead cell }} \times 100
$$

\section{E. Animals}

Male Swiss albino mice with an average bodyweight $25 \pm 5 \mathrm{~g}$ were procured from the small animal breeding station, Mannuthi, Trissur. All these animals are then allowed to live in controlled environmental conditions. The study was performed only after the clearance obtained from the institutional animal ethical committee (B110420167/MGU/SBS/IAEC/11-04-2016).

\section{F. DLA induced anticancer study}

The animals were divided into 5 groups of 6 animals each. DLA $\left(10^{6}\right)$ cells were intraperitoneally injected into the right hind limb of swiss albino mice. One set of animals was kept as normal without any treatment. EF18 was given orally to the mice for about 10 days continuously. On the basis of the acute toxicity studies, two doses of $500 \mathrm{mg} / \mathrm{kg}$ and $250 \mathrm{mg} / \mathrm{kg}$ were taken as higher and lower respectively. The standard drug used for the study was Cyclophosphamide $(25 \mathrm{mg} / \mathrm{kg})$. Parameters like tumor volume; percentage survival was evaluated up to 5 weeks. The tumor volume was measured with the help of a vernier caliper twice in a week and was calculated by using the formula $\mathrm{V}=4 / 3 \pi \mathrm{r} 12 \mathrm{r} 2$ [15]. The percentage of lifespan was calculated using the formula $\%$ ILS $=(1-\mathrm{C} / \mathrm{T}) \mathrm{X} 100)$. Where $\mathrm{T}$ is the mean survival time of the treated group and $C$ that of the control group[16] . At the end of the fifth week, the animals were sacrificed. Serum was taken for the analysis of lactate dehydrogenase (LDH) and the tumor tissue was taken and then subjected to histopathological analysis using Hematoxylin and Eosin (H \& E) staining method.

\section{G. Statistical analysis}

The entire results were expressed as the mean \pm standard deviation. The experimental groups were statistically compared by using the one- way ANOVA which is followed by Tukey's post hoc analysis. All the statistical analyses were carried out using the graph pad prism software. $P$ values $<0.005$ were considered as statistically significant. 


\section{RESULTS}

Fig.1. Images of mice bearing DLA tumors along with normal mice.

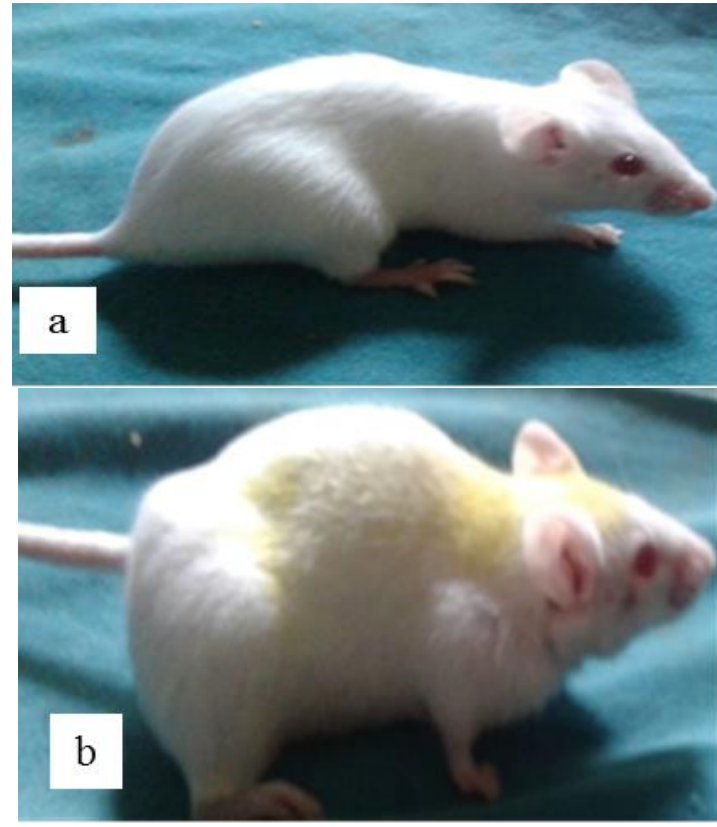

a) Normal mice b) Dalton Lymphoma Ascites induced (DLA) mice

\section{A. Cytotoxicity of EF18 on Daltons Lymphoma Ascites cells}

Cytotoxicity of EF18 was evaluated on Daltons Lymphoma Ascites (DLA) cells by short term Trypan blue exclusion method at various concentrations. As the concentration of EF18 was increased the cytotoxicity was also increased. The results are depicted in Fig 2.

Fig 2.Cytotoxicity of EF18 fraction on DLA cells at various concentrations

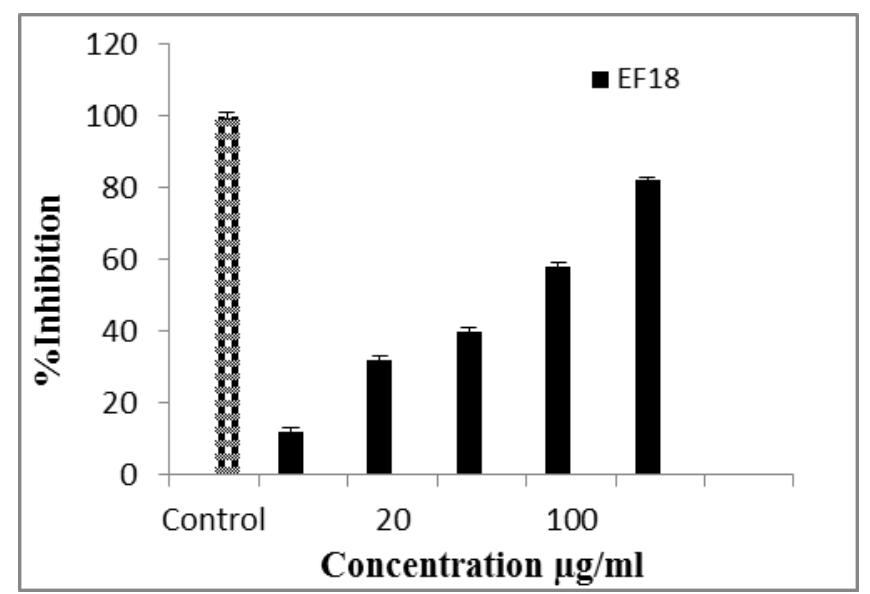

Values are the mean $\pm S D(n=3)$.

\section{B. In vivo anti-cancer studies- Effect of EF18 on Daltons Lymphoma Ascites (DLA)induced tumor volume}

Tumor volume of the DLA induced animals was evaluated in the study. Animal groups treated with EF18 especially the higher dose showed decreased tumor volume when compared to untreated DLA. Here the EF18 activity occurs in a dose-dependent manner. The effect of EF18 higher dose can be compared with that of Cyclophosphamide.

Fig 3. Effect of EF18 on DLA induced tumor volume

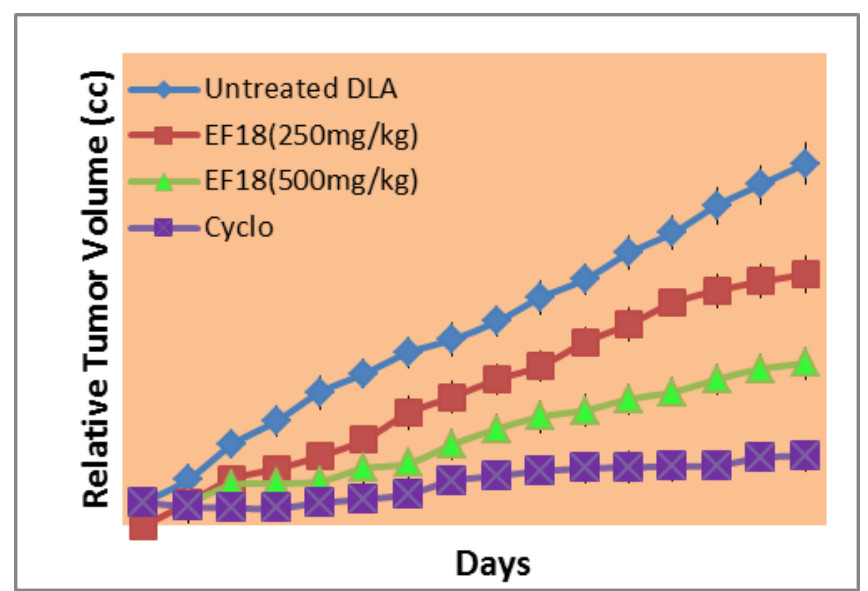

Values are the mean \pm SD $(n=3)$. Cyclo $=$ Cyclophosphamide, EF18 $(250 \mathrm{mg} / \mathrm{kg})=\mathrm{EF} 18$ lower dose,EF18 $(500 \mathrm{mg} / \mathrm{kg})=\mathrm{EF} 18$ higher dose.

\section{Effect of EF18 on percentage survival of DLA induced animal models.}

The percentage of survival of DLA induced anima groups was evaluated. Animals treated with EF 18 particularly its higher dose $(500 \mathrm{mg} / \mathrm{kg})$ exhibited increased life span than the lower dose. The activity of EF18 higher dose can be compared with that of Cyclophosphamide drugs. Results are depicted in Fig 4.

Fig 4. Effect of EF18 on percentage survival of DLA induced animal models

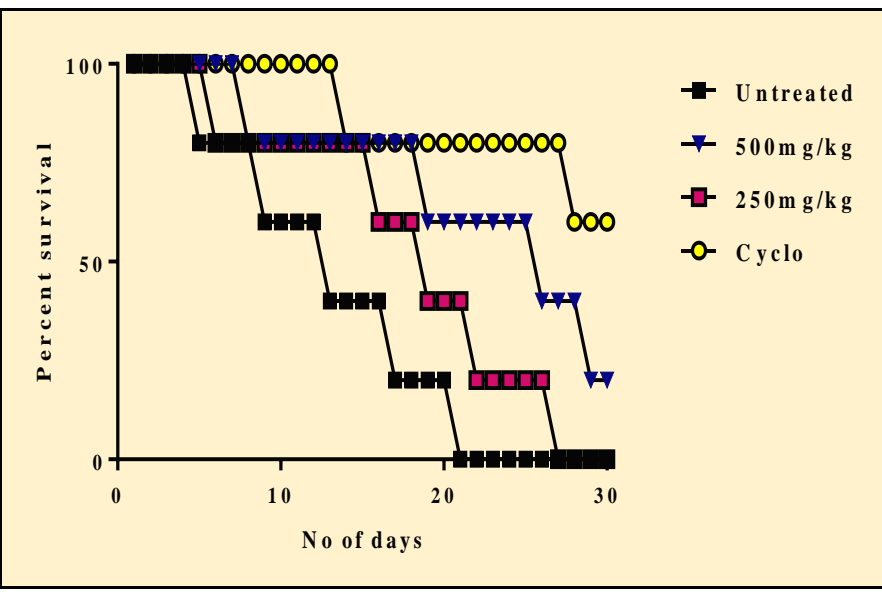

Untreated $=$ DLA control group, Cyclo=Cyclophosphamide treated group. $500 \mathrm{mg} / \mathrm{kg}=$ the higher dose of EF18, 250mg/kg=the lower dose of EF18.

\section{Effect of EF18 on Lactate Dehydrogenase (LDH) in DLA}

In cancer, the Lactate dehydrogenase (LDH) enzyme acts as a biomarker. This Lactate dehydrogenase activity was investigated in this study. Here the untreated DLA induced group act as the control. On comparing all the groups with normal groups the highest LDH activity was shown by the untreated DLA induced group. The lowest LDH activity was shown by the EF18 higher dose treated group which can be compared with that of Cyclophosphamide drug. Results are depicted in Fig 5. 
Fig 5. Effect of EF18 on LDH in DLA induced animals.

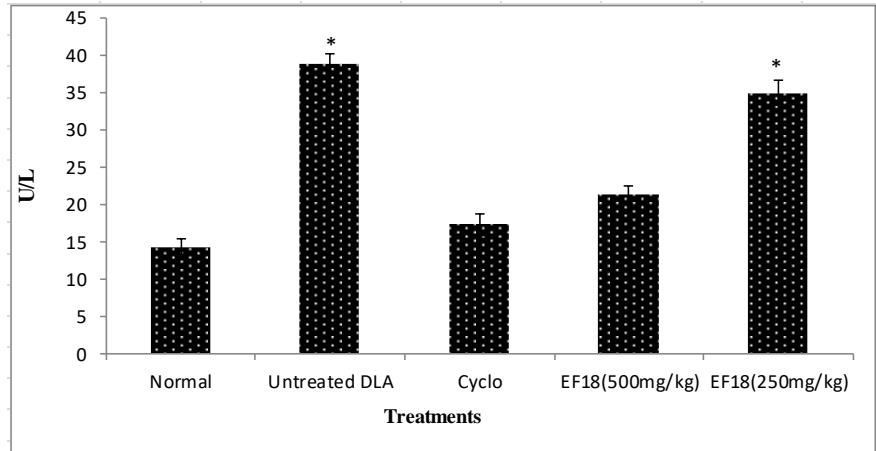

Cyclo=Cyclophosphamide treated group, Untreated DLA $=$ Control.

\section{F. Histopathological analysis of Daltons Lymphoma Ascites (DLA) induced}

Histopathological analysis of the DLA induced study was carried out. Histopathological analysis of Cyclophosphamide treated animal groups revealed that their tumor tissue composed of well discriminated neoplasmic cells that exposed vesicular nuclei having more than one or single prominent nucleoli. Numerous tumor cells exhibit more than one nucleus. The size of the tumor cell was observable.

In the untreated DLA group, well segregated neoplasmic carcinoma cells were observed on the peripheral portion of the tumor. The deeper tissue revealed dense antiinflammatory cell infiltration and necrosis. A focal collection of neoplasmic cells was detected. In EF18 lower dose $(250 \mathrm{mg} / \mathrm{kg})$ treated animals their tumor tissue showed one or more prominent nucleoli in carcinoma cells. Some tumor cells were bi nucleated and the size of the tumor cells was apparent. Necrosis and inflammation were shown by the deeper tissue of the tumor. In EF18 higher dose treated group, deeper tissues with mild inflammation and considerable mitotic activity were observed in the tumor. Results are depicted in Fig 6.

Fig 6.Histopathological analysis of DLA induced tumor tissue

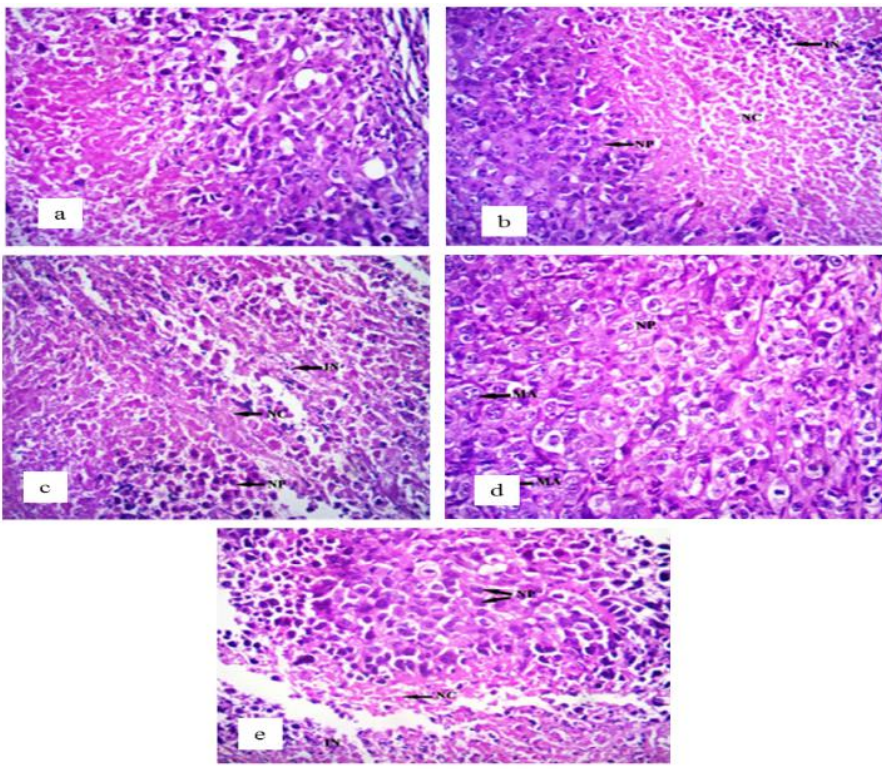

[a] normal, [b] EF18 (500mg/kg), [c]EF18(250mg/kg),

[d) $]$ Cyclo=Cyclophosphamide [e]Untreated DLA= Control.

(Here EH =Epithelial hyper keratosis, M.HK=Mild hyper keratosis, ED= Epidermis, $\mathrm{MA}=$ Mitotic activity, $\mathrm{HD}=$ Hypodermis, $\mathrm{HK}=$ Hyperkeratosis, $\mathrm{DE}=$ Dermis, $\mathrm{KL}=$ Keratin layer, $\mathrm{IN}=$ Inflammation, $\mathrm{NC}=$ Necrosis, $\mathrm{NP}=$ Nuclear pleomorphism were seen in the images)

\section{DISCUSSION}

Cancer is a series of malignant disease which affect different parts of the body. The present study deals with the in vitro and in vivo anti-cancer activity of Eugenia uniflora active fraction (EF18) on Daltons Lymphoma Ascites DLA induced animal models. Results of the short term anticancer activity of Eugenia uniflora using Trypan blue method revealed that EF18 fraction exhibited cytotoxic activity against the Dalton's lymphoma ascites cells.EF18 inhibited the growth of DLA cell lines in a dose-dependent manner.

In the current In vivo study animal groups treated with EF18 especially its higher dose reduced the tumor volume when compared to the untreated DLA group. Fig.3. illustrated the effect of EF18 on tumor volume of DLA induced animal models. The effect of EF 18 can be compared with that of Cyclophosphamide drugs. As the dosage of EF18 was increased from $250 \mathrm{mg} / \mathrm{kg}$ to $500 \mathrm{mg} / \mathrm{kg}$ the tumor volume was decreased. This revealed that EF18 exhibited anti-cancer activity in a dose-dependent manner. The percentage survival of the animal groups was increased with EF18 treatment.Fig.4. showed the effect of EF18 in the percentage survival of the DLA induced animals. On the $20^{\text {th }}$ day, the percentage survival of the animals in the untreated DLA group and EF18 lower dose was reduced than that of EF18 higher dose and Cyclophosphamide treated group. This also proved the anticancer activity of EF18 fraction.

DLA induced animal models were examined for their lactate dehydrogenase activity. The level of LDH acts as a maker in the development of cancer. Both the LDH and cancer are related. LDH level was increased in the case of cancer and its reduced level inhibits the growth of cancer. LDH supplies the energy to the cancer cells and promotes tumor initiation [17]. The LDH level of the DLA induced mice varies with that of the LDH level of normal animals. Fig.5 illustrates the effect of EF18 on LDH level. The results showed that LDH level was decreased in the case of the EF18 higher dose treated group than the untreated DLA and EF18 lower dose treated groups. This proves that EF18 exhibited anticancer activity in a dose-dependent manner. The activity of EF18 can be compared to that of Cyclophosphamide.

Histopathological analysis of DLA induced study also supports the results that EF18 exhibited anti-cancer activity in a dose-dependent manner. EF18 treatment reduced the histopathological changes and also the size of the tumor tissue as shown in the Fig.6. Basically, the Cyclophosphamide is a nitrogen mustard alkylating agent.

The outcome of the entire study suggested that Eugenia uniflora active fraction (EF18) possessed significant anticancer property in a dose-dependent manner. A substantial reduction in tumor volume and considerable increase in percentage survival was observed in the mice treated with the higher dose of EF18. Lactate dehydrogenase 
level was significantly reduced in higher dose treated groups compared to a lower dose and untreated DLA groups. Higher dose $(500 \mathrm{mg} / \mathrm{kg})$ of EF18 was better than the lower dose $(250 \mathrm{mg} / \mathrm{kg})$ of EF18 in reducing tumor volume, increasing percentage survival and reducing LDH level. The anticancer activity exhibited by the higher dose of EF18 can be compared with that of Cyclophosphamide treated groups. Phytochemical analysis of Eugenia uniflora fractions revealed the presence of various pharmacologically active phytochemicals present in it. From the outcome of the study, it was clear that the EF18 can be exploited as a virtuous source of novel drug development for cancer treatment.

\section{REFERENCES}

[1] A. M. Rahman, M.Hasan, M. A.Rahman , \& T.Afrose."Malnutrition and hiv/aids: the most threatening concerns in Bangladesh", 2018.

[2] R. S. Tagne , B. P. Telefo, J. N.Nyemb, D. M.Yemele, S. N.Njina, S. M. Goka, C., ... \& A.D.Farooq." Anticancer and antioxidant activities of methanol extracts and fractions of some Cameroonian medicinal plants", Asian Pacific journal of tropical medicine, 7 , S442-S447, 2014.

[3] A. Murali, P. Ashok, \& V. Madhavan." Antioxidant activity of leaf of Hemidesmusindicus (L.) R. Br. var. pubescens (WA) Hk. f.(Periplocaceae)-an in vivo analysis", Spatula DD, 1(2), 91100,2011 .

[4] R.Rajesh, K. Chitra, P. M.Paarakh, \& Chidambaranathan, N.” Anticancer activity of aerial parts of Aerva lanata Linn Juss ex Schult against Dalton's Ascitic Lymphoma", European Journal of Integrative Medicine, 3(3), e245-e250,2011.

[5] K.Tyagi, V.Kumar, D.Singha, K.Chandra, B. A. Laskar, S.Kundu, \& S.Chatterjee." DNA Barcoding studies on Thrips in India: Cryptic species and Species complexes", Scientific reports, 7(1), 4898, 2017.

[6] M. A. Richardson." Biopharmacologic and herbal therapies for cancer: research update from NCCAM",The Journal of nutrition, 131(11), 3037S-3040S,2001.

[7] C.Pathak, Y. K.Jaiswal, \& M. Vinayak."Modulation in the activity of lactate dehydrogenase and level of c-Myc and c-Fos by modified base queuine in cancer", Cancer biology \& therapy, 7(1), 85-91, 2008 .
[8] J. I.Anetor, G. O. Anetor, D. C.Udah, \& F.A.A. Adeniyi. "Chemical carcinogenesis and chemoprevention: scientific priority area in rapidly industrializing developing countries", African Journal of Environmental Science and Technology, 2(7), 150-156, 2008.

[9] M. L.Priya, K. B. Priya, V. S.Kotakadi, \& P. Josthna," Herbal and medicinal plants molecules towards treatment of cancer", A mini review. Am J Ethnomed, 2, 136-42, 2015

[10] I. A.Ogunwande, N. O. Olawore, O.Ekundayo, T. M.Walker, J. M. Schmidt, \& W. N. Setzer." Studies on the essential oils composition, antibacterial and cytotoxicity of Eugenia uniflora L", International journal of Aromatherapy, 15(3), 147-152, 2005.

[11] E. A.Nesy, \& L. Mathew." Studies on antimicrobial and antioxidant efficacy of Thevetia neriifolia, Juss leaf extracts against human skin pathogens" International Journal of Pharmaceutical Sciences and Drug Research, 6(2), 164-168, 2014.

[12] R. Asirvatham, A. J. M Christina, \& A. Murali." In vitro antioxidan and anticancer activity studies on Drosera indica L.(Droseraceae)", Advanced pharmaceutical bulletin, 3(1), 115,2013 .

[13] N. P.Priya, A. P.Firdous, R.Jeevana, \& K. K. Aravindakshan.' Cytotoxic and antitumor studies of acetoacetanilide N (4)-methyl (phenyl) thiosemicarbazone and its transition Metal Complexes", Indian journal of pharmaceutical sciences, 77(6), $655,2015$.

[14] Jeena, Kottarapat, V. B. Liju, \& Kuttan, Rama Dasan.” Antitumor and cytotoxic activity of ginger essential oil (Zingiber officinale Roscoe)", International Journal of Pharmacy and Pharmaceutical Sciences, 7(8), 341-344. 2015.

[15] R. Kuttan, P.Bhanumathy, K.Nirmala, \& M. C. George.” Potentia anticancer activity of turmeric (Curcuma longa)", Cancer letters, 29(2), 197-202, 1985.

[16] G. S.Ahluwalia, H. N. Jayaram, J. P. Plowman, D. A.Cooney, \& D. G. Johns."Studies on the mechanism of action of $2-\beta-d-$ ribofuranosylthiazole-4-carboxamide-V: Factors governing the response of murine tumors to tiazofurin",Biochemical pharmacology, 33(8), 1195-1203, 1984.

[17] F.Yao, T. Zhao, C. Zhong, J.Zhu, \& H.Zhao. "LDHA is necessary for the tumorigenicity of esophageal squamous cell carcinoma", Tumor Biology, 34(1), 2531, 2013 